\title{
The Issue of Current Financial Education Evaluation System
}

\section{Guo Jia}

School of Economic \& Business Management, Nanfang College of SUN-YAT-SEN University, Guangzhou, China

\section{Email address:}

badun-020@hotmail.com

\section{To cite this article:}

Guo Jia. The Issue of Current Financial Education Evaluation System. Education Journal. Vol. 4, No. 6, 2015, pp. 338-342. doi: $10.11648 /$ j.edu.20150406.13

\begin{abstract}
With the development of Chinese education reform, the independent colleges has became an effective supplement to the higher education, and it cannot be substituted for the expansion of higher education. At the same time, due to the financial talent shortage, finance program has been provided to student by most of independent colleges. However, how to establish an effective evaluation system to evaluate the education result has been faced by all colleges who provide the financial program. This paper would like to discuss the current financial education evaluation system, and study an effective financial education evaluation system.
\end{abstract}

Keywords: Financial Education, Evaluation System, Education Evaluation

\section{浅谈独立学院金融学科教学评价体系的完善}

\section{郭佳}

中山大学南方学院, 经济学与商务管理系, 广州, 中国

\section{邮箱}

badun-020@hotmail.com

\begin{abstract}
摘要: 随着中国教育改革的不断推进, 独立学院作为公办本科院校的有效补充, 对高等教育扩招起到了不可替代的作 用。同时，金融人才特别是高端的金融人才最为当前市场急需的资源，被作为热门专业并被独立学院广泛开设。但是 在各院校广泛的开设金融类课程的同时，如何能够培养出符合市场需求的金融类人才成为在专业建设中的首要问题， 而合格人才的标准就转移到了相关学科的教学评价体系中来。本文希望通过对广州地区多家开展金融教学的高校教学 评价体系的调查, 探讨现有金融教学中的评价体系的优劣, 同时提出改进教学评价体系的建议。
\end{abstract}

关键词：金融教学, 评价体系, 教学评估

\section{1. 引言}

随着中国教育改革的不断推进, 独立学院作为公办 本科院校的有效补充, 对高等教育扩招起到了不可替代 的作用。同时，高端的金融人才作为当前市场急需的资 源, 也被作为热门专业在独立学院中广泛开设。但是在 各院校广泛的开设金融类课程的同时, 如何能够培养出 符合市场需求的金融类人才成为在专业建设中的首要
问题, 而合格人才的标准就转移到了相关学科的教学评 价体系中来。对于独立学院而言, 结合独立学院自身的 特点与学生的整体接受能力来看, 很多独立学院提出了 与传统科研型大学差异化的人才培养方案, 具体来说就 是培养实用型的金融人才。而传统科研型的大学因其自 身更多着重于科研建设的发展, 究其评价体系而言也更 多的关注于学生对传统概念的理解与创新运用, 而没有 更多的关注学生对于所学相关知识的运用能力。简单来 
说既对学生动手能力的考查并不强。而对于独立学院为 了差异化培养而言, 显然这样的教学评价体系在某种程 度上是不能满足其对学生进行实用型人才培养的需要 的。因此, 建立一套适合独立学院层次学校对金融相关 学科的教学评价体系对于相关院校的人才培养将起到 至关重要的作用。

\section{2. 现有金融学科教学评价体系及其问题}

经过相关院校多年对于金融专业的建设与实践, 摸索 出了一套现有的对于金融专业学科的教学评价系统。这其 中主要包括考试、学业论文、实践报告和学生演讲等。为 此, 对广州地区的多所高校进行了相关抽样调查, 成功取 得数据的高校共 6 所, 其中传统科研型院校 3 所, 独立学院 3 所。调查中的6所高校均开设了金融类课程, 6所高校对 相关专业开设的专业课平均为50门。调查分别对各高校采 取学科考试、学业论文、实践报告等三种学科考查手段的 数量进行了统计, 数据见下表 1 。

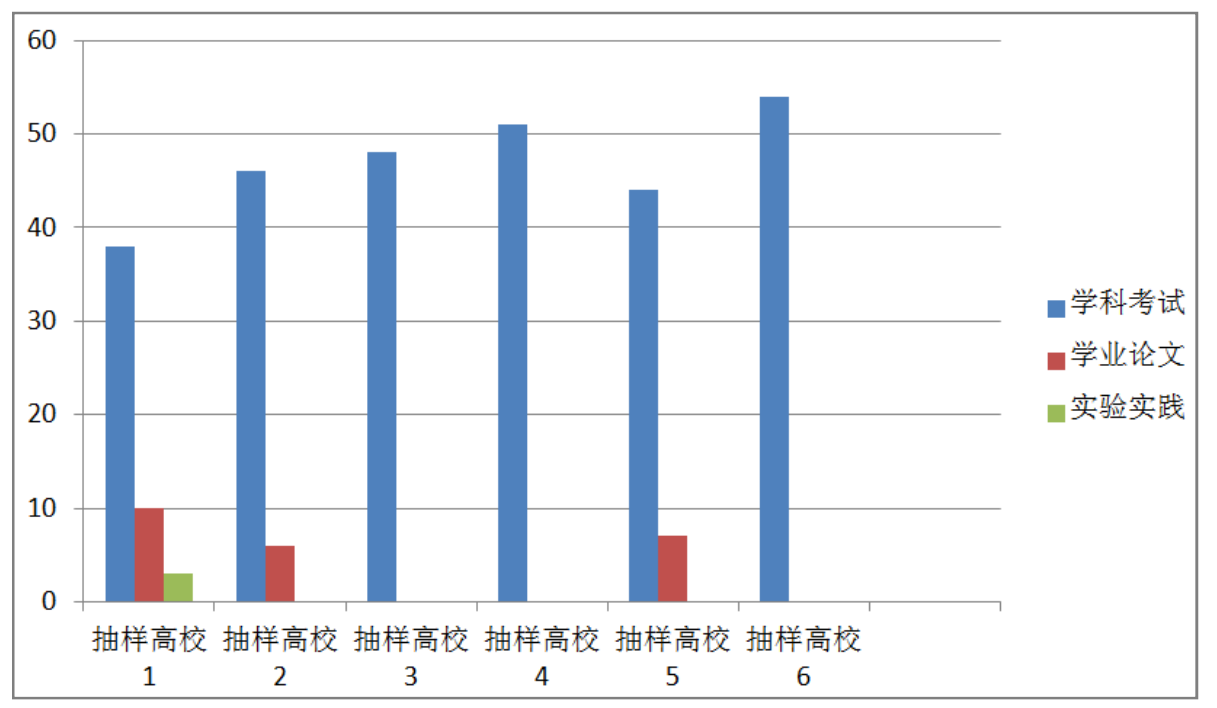

表1 学科考查手段统计。

基于调查数据, 逐一对相关内容进行总结与分析

\section{1. 学科考试}

学科考试作为传统的教学评价手段目前被广泛的运 用于各学科的教学评价中。学科考试具有众多的优点, 主 要有能考查学生对相关知识的记忆程度; 拥有相对统一的 评价标准; 对于任课老师而言考核形式简单, 可操作性强 等优点。
经调查显示, 在成功获得调查数据的 6 间高校中, 全 部6所高校都采取了以考试作为课程的评价手段。其中5 家高校以考试作为教学评价手段的比例占全部需评价课 程总数的 $80 \%$ 以上, 占总调查比例的 $83 \%$ 以上, 剩余 1 间高 校采用考试手段进行评价课程的比例也占到了总开设课 程的 $70 \%$ 以上。其中独立学院层次以学科考试为主要考察 手段的占比均超过 $80 \%$, 当中的一所高校学科考试所占比 重甚至高达 $94 \%$ 。传统科研型大学中仅有一家以学科考试 作为评价方式的比重低于 $80 \%$ 。见表2。

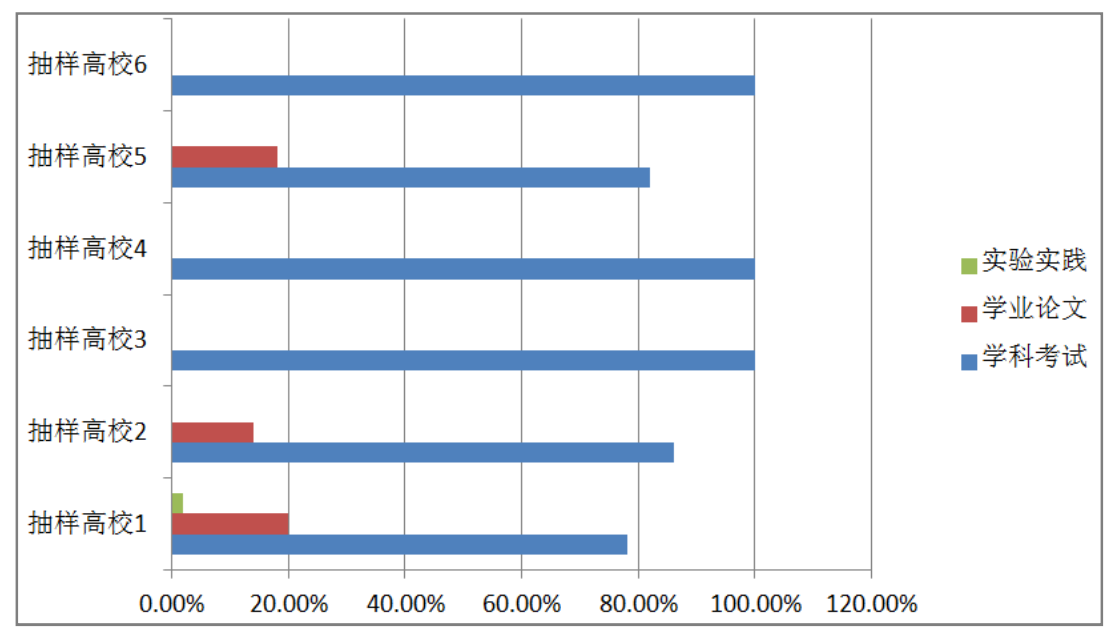

表2 考查方式所占比例。 
由此可见学科考试是高校中对金融类课程教学成果 评价的最主要手段。但同时值得指出的是, 学科考试的方 式虽然能更好的显示学生对所学理论知识的记忆能力, 但 是其局限性也是显而易见的。其中最明显的不足是学科考 试的方式不能很好的考查学生对于所学知识的运用能力 和动手能力, 而这些能力恰恰又是独立学院在培养相关人 才中与传统科研型大学差异化发展的方向。因此从调查所 显示的数据来看, 独立学院对于教学评价的方式可能未能 体现出其对学生进行实用型人才培养的目标, 反而传统科 研型大学在学科考察中运用了更多其他的考查手段并且 可能更能够体现学生的整体综合素质。

\section{2. 学业论文}

除学科考试外, 学业论文正逐渐成为教师考查学生对 本学科所学知识的重要考查手段之一。相对学科考试而言, 学业论文更具灵活性, 同时能够通过设置更多条件与背景 综合的考查学生对所学知识的掌握与理解水平, 并且也会 避免学生在考前只要通过短时间突击, 死记硬背就能通过 考试的情况, 因此能够更全面的了解学生对所学知识理解 运用的水平, 并及时掌握教学中的不足与缺陷。

同样基于前文所提的相关调查我们发现, 在调查样本 的6所高校中, 有3所高校的学科结业形式不包括学业论文 方式, 占总调查比例额 $50 \%$ 。其中, 两所传统科研型大学 和一所独立学院采取了学业论文的评价方式对教学成果 进行测评。通过进一步的调查我们发现, 在不采取学业论 文的 3 所高校中, 有 $43 \%$ 的受调查教师认为不采取学业论文 的原因是学业论文需要花费大量时间精力进行批改, 无形 中增加了教师的工作量; 另有 $47 \%$ 的教师认为相关教务部 门的评价体系约束了教师通过学业论文的方式对学生进 行考核; 更为有趣的是, 有 $23 \%$ 的被访教师认为传统的考 试观念制约了他们通过学业论文对学生考查的动力。

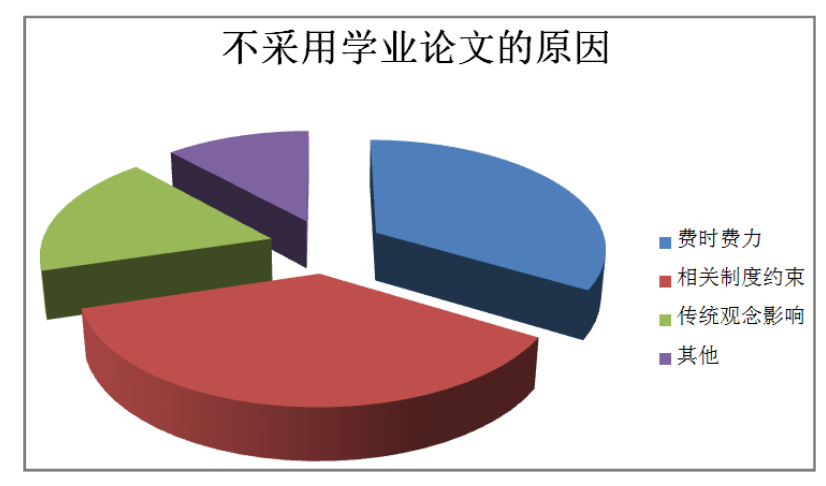

表3 排斥学业论文原因。

然而, 相对于中小学的学科考查而言, 大学课程尤其 是经济金融类课程更注重学生对所学知识理解应用的能 力, 单一通过考试的手段更可能不能更好的考查学生对相 关知识的掌握程度。而学业论文是一种更为有效而全面考 查学生对所学相关知识的考查手段, 通过学业论文, 任课 老师既可以考查学生对相关基础知识的掌握程度, 同时也 可以通过文章中的观点和意见了解学生对相关知识的应
用于创新能力。但从调查的结果来看, 现有高校运用学业 论文作为学科考核的比例还有待提高。这里既有传统教育 观念的转变, 也有对老师提出更高要求的需要。同时学生 也需要适应新的考查手段, 而且必须指出的是, 以学业论 文为考查手段时, 相关任课教师必须严把学生的抄袭率。 尤其在网络化发达的今天, 学生普遍存在网络抄袭的习惯, 而这种习惯对今后的工作学习是有极大危害的。因此任课 教师的从严把关是强化学业论文考核基础。

\section{3. 实践实验}

除了学科考试与学业论文外, 实践实验也成为考查学 生对相关专业知识掌握的一种重要手段。对于金融类专业 课程尤其是独立学院的金融类人才培养而言, 除理论外, 动手实践能力更为重要。而考查学生的动手实践能力仅凭 学科考试等手段是不可能有效完成的。因此, 通过实践实 验手段进行考核就变得尤为重要。

还是通过前文中所提到的调查, 在被调查的广州地区 六所高校中, 仅有一间传统公立研究性大学采用了实验考 查手段。这说明实验考查这种手段在目前的高校金融类课 程考核体系中所占比重较低, 且并未被广大高校所普遍接 受。究其愿意笔者认为有三。第一, 传统的学科考查思维。 传统的学科考查思维认为, 对学生掌握知识的考查最为有 效的手段就是考试。所谓考考考, 老师的法宝就是这个理 论的重要体现。第二, 考查硬件设施无法跟上。采用实验 考查就要求相关学科拥有完善的实验教学设备与教学工 具。以金融类课程的 “国际结算” 为例, 课程中需学生掌 握商业银行的各种结算工具与相关交易系统如SWIFT系统 等, 这类知识虽然通过传统的考试可以考察学生对相关理 论的掌握情况, 然而要了解学生对其具体操作的能力, 如 对SWIFT系统的掌握程度, 则最好通过实践实验的考查手 段, 而采取这种考查手段就需要相关课程拥有完善的实验 室软硬件设备设施。而在所调查的几所高校中, 相关的实 验设施相对是不够完善的, 这就从客观上制约了采用实践 实验考核的途径。第三。相关教师缺乏对实践教学的经验。 采用实践教学除了要求拥有相关的软硬件设施外, 另一重 要的因素是教师水平的提高。目前高校中的教师普遍拥有 硕士博士学位, 设置博士后也大有人在, 然而学位的高低 更多的只能体现出对理论的理解, 而相关的实践未必拥有 更多的经验。因此, 这就需要相关课程的老师除了对学所 理论知识的掌握外还需要提高自身的实践动手能力, 从而 才能进一步提高教学能力, 而这一点对于独立学院类的大 专院校而言就更显得更加尤为重要。

\section{3. 提高金融学科教学评价体系的意见与建议}

\section{1. 加大非考试类考查在学业考查中所占的比重}

高等学校尤其是独立学院层次高等学校的金融类课 程更加注重于传统科研型大学的差异化发展, 因此培养学 生的实践动手能力就变得尤为重要。加大通过学业论文、 实践实验等的方式对学生进行学科考查, 有助于考查学生 对所学知识的动手运用能力, 从而实现差异化培养人才的 
目标。因此, 转变传统通过考试考核学生的考核思想尤为 重要, 这就要求任课教师明确对人才培养最本质目标的认 识, 充分意识到培养学生对所学金融类相关知识的运用能 力提高, 同时对教师自身也应提出更高的要求。

通过前文的调查数据可知, 目前高等院校对于金融类 学科的考核方式主要还以考试为主。但对于差异化培养金 融类人才的角度, 单一以考试的方式评价学生显然是不能 够满足差异化培养的需求的。因此建议适当加入更多其他 的考核手段, 具体方式如下:

\subsection{1. 实践报告形式}

金融学科与理工科在某些具体学科中有相似之处, 也 需要学生拥有较强的动手能力, 而这种能力的考核但从考 试的手段是无法全面的考查的, 运用实践报告的形式, 要 求学生对所学知识亲自动手实践, 之后做出实践报告, 可 以有效考查学生掌握知识的水平和动手能力的水平。

\subsection{2. 社会实践形式}

金融学作为一门社会学学科, 需要学生进行充分的 社会实践, 例如个人理财的课程, 课堂的讲授只能向学 生说明相关的基础知识, 然而对于学生来说, 真正掌握 课程的所有知识就需要有充分的社会实践。因此, 在日 常教学中考查中, 加入社会实践的考查内容, 有助于对 学生全面掌握所学知识进行有效的考查, 具体可以要求 学生填写完整的社会实践构思、实践过程报告与实践结 果报告的形式进行。

\section{1. 3. 通过相关实验室进行考核}

对于应用型金融专业本科生来说, 动手能力是有别于 传统学术型本科生的重要标志。因此, 部分课程如国际结 算、商业银行经营等动手性较强的课程, 可以通过相关实 验室操作对学生进行考核。例如, 在国际结算的课程中, 目前大部分高校都建有相关实验室和实训软件, 可以充分 利用这些资源, 以实际操作的手段, 如要求学生填写完整 的SWIFT报文等形式对学生进行考察。

通过以上这些手段, 结合传统的考试和学业论文等形 式, 相信可以对学生进行更为全面的考查。

\section{2. 加强相关实验室的软硬件建设}

对于金融类学科而言, 实践考察是考量学生实际动手 能力的一种重要手段, 运用实践考察的一个先决条件则是 建立完整的教学实验体系和拥有完善的实验室软硬件设 备。目前, 大部分高校都拥有较为完善的学科实验室, 如 模拟银行等。但这些实验室相对独立, 且用途相对单一, 更多的之所作为某一特定课程的一种延伸。如果需要对学 生进行综合的实践考察, 则单一目的的实验室是无法满足 对学生整体综合素质考察的需求的。这就要求各高校在不 断完善现有实验室建设的基础上, 建立成套的实验室体系, 通过实验室建设将个相关学科有机的结合在一起, 扩展实 验室的用途。这样才能让学生在实验教学中做到融会贯通, 即培养了学生整体的认知能力, 同时也培养了学生对课程 体系的动手能力与理解水平。具体而言, 高等院校应当对 现有的各金融类实验室指派一致两名专职负责实验室建
设的教师, 负责将各独立的实验项目或实验室整合成一个 整体。例如。金融类专业经常会建立的单证实验中心可能 用于国际结算等课程实践、报关实验中心用通关实务等课 程实践; 模拟银行用于商业银行经营等课程的实践; 通过 整合可以将这些实验室或实验中心组成一个金融单证实 验体系, 即使得学生理解和掌握单一课程的实践内容, 同 时也帮助学生通过实验体系对相关课程之间的关联性得 到充分的了解和实践。

\section{4. 结论}

在金融学不断被重视的今天, 高等院校中的金融类课 程也越来越受到重视, 实用性与应用型的金融类人才也越 来越受到用人单位的欢迎。这就要求各高校在培养金融类 人才的过程中不断的适应市场对人才的需求。如何评价应 用型的人才就显得更为重要。通过本文的论述, 我们可以 发现, 传统的人才评价的手段更多的注重于对基础理论知 识的掌握和记忆, 而新时期的金融类人才更多的需要对所 学知识的实践运用与动手能力, 因此传统的人才评价体系 是无法满足当代市场与金融机构对人才培养的需求的。这 就要求作为人才培养的高等院校改变现有对人才评价的 模式与体系。而改革的目标应该是体系化的评价, 即不单 一的通过某一或两种手段去评价学生的整体素质。理想的 人才评价体系应是通过多种途径与手段全方位的考查一 个学生对包括基础理论、理论运用、实际操作、理论创新 等多方面综合素质的能力。最后, 通过本文, 希望能对相 关高校在人才培养评价体系建设研究方面做出初步的探 索, 并希望今后相关院校能够建立一套更为完善的金融类 人才培养体系。

\section{参考文献}

[1] 郑延福, 《本科高校教师教学质量评价研究》, 北京, 科 学与教育管理, 2012。

[2] 任艳红, 《高校教学评价制度的反思与重构》, 北京, 教 育学原理, 2014。

[3] 韦剑剑, 《本科教学评价的后现代主义反思》, 北京, 高 等教育学, 2014。

[4] 余远富, 王庆仁, 《高校研究性教学评价体系的构建》, 上海, 高校工程教育研究, 2013。

[5] 张静, 《试论高校双语教学评价体系的构建》, 北京, 高 教探索, 2014。

[6] 孙忠梅, 《地方高校实验教学评价体系的研究与实践—— 以深圳大学为例》, 哈尔滨, 黑龙江教育学院学报, 2013。

[7] 朱元胜, 《实践教学评价体系构建探索与实践》, 杭州, 浙江纺织技术学院学报, 2007。

[8] 刘敏, 促进学生发展的教学评价体系, 上海, 上海师范大 学, 2008。 
[9] 董江红, 高等职业教育实践课堂教学评价质量评价体系研 究, 西安, 西安建筑科技大学, 2008。
[10] 章晓辉、陈再萍, 高校课堂教学质量的有效教学评价体系 结构研究, 北京, 现代教育科学, 2008。 